Interfaces and Free Boundaries 17 (2015), 263-288

DOI $10.4171 / \mathrm{IFB} / 342$

\title{
The mesa problem for the fractional porous medium equation
}

\author{
JUAN LUIS VÁZQUEZ \\ Departamento de Matemáticas, Universidad Autónoma de Madrid, 28049 Madrid, Spain \\ E-mail: juanluis.vazquez@uam.es
}

[Received 13 July 2014 and in revised form 10 April 2015]

We investigate the behaviour of the solutions $u_{m}(x, t)$ of the fractional porous medium equation

$$
u_{t}+(-\Delta)^{s}\left(u^{m}\right)=0, \quad x \in \mathbb{R}^{N}, t>0 .
$$

with initial data $u(x, 0) \geqslant 0, x \in \mathbb{R}^{N}$, in the limit $m \rightarrow \infty$ with fixed $s \in(0,1)$. We first identify the limit $F_{\infty}$ of the Barenblatt solutions $U_{m}(x, t)$ as the solution of a stationary fractional obstacle problem, and we observe that, contrary to the case $s=1$, the limit is not compactly supported but exhibits a typical fractional tail with power-like decay. In other words, we do not get a plain mesa in the limit, but a mesa with a tail. This is not the whole story since the limit of $V_{m}(x, t)=m t U_{m}^{m}(x, t)$ exists and is compactly supported (in $x$ ). We then study the limit $m \rightarrow \infty$ for a wide class of solutions with nonnegative initial data, and show also in this setting the phenomenon of initial discontinuity, whereby the solution does not take on the prescribed initial data. Finally, we derive counterexamples to expected propagation and comparison properties based on symmetrization and pose a related open problem.

2010 Mathematics Subject Classification: Primary 35K65, 35S10, 26A33, 76S05

Keywords: Nonlinear fractional diffusion; fundamental solutions; singular limit; mesa profile; obstacle problem; symmetrization

\section{The mesa problem as limit of the FPME when $m \rightarrow \infty$}

This paper deals with the limit $m \rightarrow \infty$ of the solutions of the family of fractional porous medium equations (FPME) of the form

$$
u_{t}+(-\Delta)^{s}\left(u^{m}\right)=0, \quad x \in \mathbb{R}^{N}, t>0 .
$$

Here $(-\Delta)^{s}$ is the fractional Laplacian operator with $0<s<1$. We will take initial data

$$
u(x, 0)=u_{0}(x),
$$

where $u_{0}$ is a nonnegative and integrable real function defined in $\mathbb{R}^{N}$, or a Dirac delta. Under these assumptions the Cauchy Problem produces a unique solution $u_{m}(x, t)$ for every finite $m>1$ and every $0<s<1$; cf. [30, 31] for the basic theory and the survey paper [52] for recent progress in the qualitative analysis. As $s \rightarrow 1$ with $m>1$ fixed we obtain in the limit the standard porous medium equation (PME), $u_{t}=\Delta u^{m}$, whose mathematical theory and applications are by now well-known, see [48]. We may even take a Dirac delta as initial data, and then the solutions are called fundamental solutions or Barenblatt solutions; in the fractional case the Barenblatt solutions have been constructed in [51] where their uniqueness, properties, and their role in the asymptotic behaviour are explained, see Section 3 below. 
The study of the differences in the behaviour of diffusion equations involving the standard Laplacian, i.e., involving a local operator, and the fractional variants, which involve nonlocal diffusion operators representing long-range interactions, is a matter of much current attention. The comparison between the PME and the FPME is a convenient setting for such analysis. We tackle here the limit $m \rightarrow \infty$ in the latter model and compare it with what is known in the first.

Indeed, the dramatic effect of the limit $m \rightarrow \infty$ is well known in the PME case, i. e., equation (1.1) with $s=1$, as described in $[3,8,9,15,23,26,27,29,36]$ and other related literature. It is proved that given a nonnegative initial datum $u_{0}(x) \in L^{1}\left(\mathbb{R}^{N}\right)$ there is a unique weak solution $u_{m}(x, t)$ of the PME defined in $Q=\mathbb{R}^{N} \times(0, \infty)$ that is uniformly bounded and $C^{\alpha}$ continuous for $t \geqslant s>0$. If $u_{0}$ is compactly supported, so is $u_{m}(\cdot, t)$ for every $m>1$ and every $t>0$. Concerning the limit, it is proved in the above references that there exists a limit of the family of solutions $\left\{u_{m}(x, t): m>1\right\}$ as $m \rightarrow \infty$, and this limit is a function $u_{\infty}(x)$ that does not depend on time, so that no diffusion occurs at all for $t>0$. If the initial function satisfies the bounds $0 \leqslant u_{0}(x) \leqslant 1$, then we also have

$$
\lim _{m \rightarrow \infty} u_{m}(x, t)=u_{0}(x),
$$

hence a completely stationary situation. The more interesting case happens when $u_{0}$ is larger than 1 on a nontrivial set, and in that case there still exists a unique limit

$$
u_{\infty}(x)=\lim _{m \rightarrow \infty} u_{m}(x, t) \quad \text { and } \quad 0 \leqslant u_{\infty}(x) \leqslant 1 .
$$

This means that the upper part of the initial datum collapses at $t=0+$ to the level $u=1$, in response to the fact that the diffusivity $m u^{m-1} \rightarrow \infty$ whenever $u>1$. In other words, we are facing a singular perturbation limit and there is an associated initial discontinuity layer. Describing such phenomenon is the content of the mathematical theory of the mesa problem. A brief summary is as follows: the upper level set $\Omega=\left\{x: u_{\infty}(x)=1\right\}$ is found by solving a certain variational inequality, in other words, an obstacle problem, while away from $\Omega$ we have $u_{\infty}(x)=u_{0}(x)<1$ (no diffusion takes place there).

The name of mesa problem for this problem comes from the typical 'mesa shape' of $u_{\infty}(x)$ (the shapes seen in landscapes in the West of the USA). What makes the analysis more interesting is the numerically observed fact that the mesa formation is already apparent for relatively low values of $m$, say $m \approx 6$, using typically bell-shaped initial data, cf. [23]. It is worth mentioning that $\Omega$ can be much larger than the set where $u_{0}(x)>1$. This happens because of the collapse of the 'mountain' represented by the part where $u_{0}(x)>1$. In the special case in which we take as initial data a Dirac delta, the set $\Omega$ is just a ball $B_{R}(0)$, and $u_{\infty}(x)=0$ for $|x|>R$.

The fractional case. We study here the limit $m \rightarrow \infty$ in the case of fractional diffusion, $0<s<1$. The analysis shows some common features with the previous picture, but also quite interesting novelties which are worth describing. We will examine in detail some of these novel aspects. First of all, we focus on the limit behavior of the Barenblatt solutions since this family plays a major role in the analysis of the standard mesa problem. We recall that these solutions have the form [51]

$$
U_{m}(x, t)=t^{-N \beta_{m}} F_{m}\left(x t^{-\beta_{m}}\right), \quad \beta_{m}=1 /(N(m-1)+2 s) .
$$

Here we will identify the limit $m \rightarrow \infty$ of these solutions, which is a nontrivial task since the profiles $F_{m}$ are not explicit and the limit is highly singular. We observe that, contrary to the case $s=1$, the limit $u_{\infty}(x, t)=F_{\infty}(x)$ is not compactly supported but exhibits a typical tail with 
power-like decay at infinity. In other words, we do not get a plain mesa in the limit but a mesa with a tail. Describing the coincidence set where $F_{\infty}(x)=1$ as well as the behaviour of the tail are main issues that we want to address. Here is the first result.

THEOREM 1.1 As $m \rightarrow \infty$ we have for all $x \in \mathbb{R}^{N}$ and $t>0$

$$
\lim _{m \rightarrow \infty} U_{m}(x, t)=F_{\infty}(x),
$$

where $F_{\infty}$ is radially symmetric function, non-increasing in $r=|x|, 0 \leqslant F_{\infty}(r) \leqslant 1$, and $\int F_{\infty}(x) d x=1$. Moreover, there exists an $R>0$ such that $F_{\infty}(r)=1$ for $0 \leqslant r \leqslant R$ while $0<F_{\infty}(r)<1$ for $r>R$.

The proof is given in Section 4. The analysis of the limit uses heavily a pair of associated functions, namely $U_{m}(x, t)$ and

$$
W_{m}(x, t)=m U_{m}^{m}(x, t)=t^{-m \alpha} G_{m}\left(x t^{-\beta}\right),
$$

where $G_{m}=m F_{m}^{m}$. Both $U_{m}$ and $W_{m}$ (or equivalently, $F_{m}$ and $G_{m}$ ) behave very differently for large values of $m$. In the next step we are able to identify both the level set $F_{\infty}=1$ of the limit and the tail via the solution of an obstacle problem.

THEOREM 1.2 Let us consider the following obstacle problem for two variables $P$ and $G$ : Given the obstacle $\Phi(x):=C-\left(|x|^{2} / 2 N\right)$ with $C>0$, to find nonnegative radial functions $P$ and $G$ such that

$$
\left\{\begin{array}{l}
(-\Delta)^{1-s} P(x)=G(x), \\
G(x)(P(x)-\Phi(x))=0, \\
G(x) \geqslant 0, \quad P(x)-\Phi(x) \geqslant 0,
\end{array}\right.
$$

and $P(x) \rightarrow 0$ as $|x| \rightarrow \infty$. This problem has a unique solution for every $C>0$, and $P \in$ $C^{2-s}\left(\mathbb{R}^{N}\right), G \in C^{s}\left(\mathbb{R}^{N}\right)$. Then,

(i) $\quad F_{\infty}$ of the previous theorem is given by $F_{\infty}(r)=N P^{\prime}(r) / r$. See the more precise formula (5.11). The condition $\int F_{\infty}(x) d x=1$ allows to determine the exact value of the constant $C$ by a scaling argument.

(ii) Once $C$ is chosen, the limit function $G_{\infty}=\lim _{m \rightarrow \infty} G_{m}$ coincides with the solution $G$ of this obstacle problem. It is supported in the ball $B_{R}(0)$, with $R$ as in Theorem 1.1, and it has the explicit form: $G_{\infty}(x)=c\left(R^{2}-|x|^{2}\right)^{s}$ for some $c>0$.

(iii) $F_{\infty}(x)$ decays like $c|x|^{-(N+2 s)}$ as $|x| \rightarrow \infty$.

This is proved in Section 5, where full details are given. Note that (1.4) is the fractional obstacle problem studied by Caffarelli et al. in [37], [16], [1]. This leads to another surprise: the limit of $G_{m}$ as $m \rightarrow \infty$ is just the spatial profile of the self-similar solution that describes the asymptotic behaviour of a quite different nonlinear fractional diffusion model, namely

$$
u_{t}=\nabla \cdot\left(u \nabla(-\Delta)^{-s} u\right),
$$

studied by Caffarelli and Vázquez in [18], see also Biler et al. [10-12]. This seems quite unexpected, but subsequent work with Stan and Teso [38, 39] shows that it is part of a more general correspondence between different models of nonlinear nonlocal diffusion of fractional type. 
Once this analysis is done, we devote Section 6 to identify the limit for a class of nonnegative and integrable initial data. In the PME case, it has been proved that there exists a unique limit $u_{\infty}(x)=\lim _{m \rightarrow \infty} u_{m}(x, t)$, and $0 \leqslant u_{\infty}(x) \leqslant 1$. A convenient variational inequality identifies the indicator function $w_{\infty}(x)$ that in turn determines the limit function $u_{\infty}$. In the fractional case we will have a mesa limit with tails, and these exhibit a fractional type decay as $|x| \rightarrow \infty$. Our present analysis of the fractional case with general data is only partial, a complete analysis of fractional mesa problems is being done separately by Quirós and the author [34]. But the results we present here are illuminating since they show another difference with the standard PME: it is generally false that the limit functions $u_{\infty}(x)$ equal $u_{0}(x)$ at the points where $u_{\infty}(x)<1$.

This analysis is also needed in order to present a final contribution of our study. Thus, in Section 7 we obtain a contradiction with the standard result of the symmetrization theory (based on concentration comparison) that is known to be true for the standard porous medium equation, cf. [45], [47]. This is another remarkable difference between standard and fractional diffusion; such a failure of comparison was first demonstrated by Volzone and the author in [54] by completely different methods.

Notations. $\quad B_{r}(x)$ denotes the open ball in $\mathbb{R}^{N}$ with center $x$ and radius $r>0$, and $\omega_{N}$ denotes the volume of the unit ball in $\mathbb{R}^{N}$. The $s$-Laplacian operator $(-\Delta)^{s}, 0<s<1$, acting in $\mathbb{R}^{N}$ is precisely defined in the literature, cf. [32, 40, 50] among the many references. We will write $s^{\prime}=1-s$. We will have to keep track of the delicate dependence of a number of constants on the values of $m \gg 1$, but we will often use the same letter $C$ for different positive constants when their value is not important in the context. The dependence on $s$ will not be important in most of the text as long as $0<s<1$.

\section{Limits of Barenblatt solutions for the standard PME}

We re-do the analysis of the known case $s=1$ in order to introduce some detailed calculations that will fix ideas, and serve as precedent and motivation. Actually, the situation for the standard porous medium equation

$$
u_{t}=\Delta\left(u^{m}\right), \quad m>1
$$

posed in the whole space $x \in \mathbb{R}^{N}, N \geqslant 1$, has been discussed in the literature. Thus, we have the following explicit formulas for the fundamental solution of the PME with data $U_{m}(x, 0)=M \delta(x)$ :

$$
U_{m}(x, t)=t^{-\alpha} F(\xi), \quad F_{m}(\xi)=\left(C-k \xi^{2}\right)_{+}^{1 /(m-1)}
$$

where $\xi=x / t^{\beta}$ and

$$
\alpha=\frac{N}{N(m-1)+2}, \quad \beta=\frac{\alpha}{N}, \quad k=\frac{(m-1) \alpha}{2 N m} .
$$

Let us go into some quantitative details that are important to pass to the singular limit. Here the free constant $C>0$ is related to the mass $M$ by the formula

$$
M=d_{m} C^{\gamma_{m}}, \quad \gamma_{m}=\frac{N}{2(m-1) \alpha},
$$


where $d_{m}$ is given by the formula

$$
d_{m}=N \omega_{N} \int_{0}^{\infty}\left(1-k|y|^{2}\right)^{1 /(m-1)} y^{N-1} d y=N \omega_{N} k^{-N / 2} \int_{0}^{\infty}\left(1-|y|^{2}\right)^{1 /(m-1)} y^{N-1} d y .
$$

Actually, $d_{m}$ depends also on the dimension $N$ but since this dependence does not play a role here we will omit it as a rule. In the same we write $\alpha_{m}, \beta_{m}, \gamma_{m}$, and so on.

We can now pass to the limit $m \rightarrow \infty$. We have $m \alpha_{m} \rightarrow 1, \gamma_{m} \rightarrow N / 2$, both nontrivial limits; but $k_{m} \sim 1 /(2 N m) \rightarrow 0$, so we rescale $C=\widehat{C} / m$ and with

$$
\widehat{d}_{m}=\frac{d_{m}}{m^{\gamma}}=m^{-\gamma} N \omega_{N} k^{-N / 2} \int_{0}^{\infty}\left(1-|y|^{2}\right)^{1 /(m-1)} y^{N-1} d y \rightarrow \omega_{N}(2 N)^{N / 2}:=D_{\infty} .
$$

Note that $M=\widehat{d}_{m} \widehat{C}^{\gamma}$. Using this, we easily conclude that

$$
\lim _{m \rightarrow \infty} m\left(U_{m}\right)^{m}(x, t)=W_{\infty}(x, t r):=\frac{1}{2 N t}\left(\left(\frac{M}{\omega_{N}}\right)^{2 / N}-|x|^{2}\right)_{+}=\frac{1}{2 N t}\left(R_{0}^{2}-|x|^{2}\right)_{+}
$$

and

$$
\lim _{m \rightarrow \infty} U_{m}(x, t)=U_{\infty}(x):=\chi_{B_{R_{0}}(0)}(x) .
$$

We have put $M=\omega_{N} R_{0}^{N}$, and this is easily calculated using the fact that $U_{\infty}=1$ whenever $W_{\infty}>0$.

○ Note that $\Delta_{x} W_{\infty}(x, t)=-1 / t$ in the set $\left\{(x, t): W_{\infty}>0\right\}$, which is the exact limit of the well-known a priori estimate:

$$
\Delta U_{m}^{m-1}=-\frac{(m-1) \alpha}{m t},
$$

which holds in the same type of positivity set, $\left\{U_{m}>0\right\}$, for finite $m>1$.

○ It is interesting to write the equation for $w_{m}=m\left(u_{m}\right)^{m}$, since it allows us to capture part of the information about the singular limit $m \rightarrow \infty$. The equation is

$$
w_{t}=m^{1 / m} w^{1-(1 / m)} \Delta w
$$

In the limit $m \rightarrow \infty$ we have $w_{m} \rightarrow w$ and the equation becomes $w_{t}=w \Delta w$. This equation has $W_{\infty}(x, t)$ as radial separable-variables solution, with free parameter $R_{0}>0$.

○ For the self-similar profile we have the following limits as $m \rightarrow \infty$ :

$$
F_{m}(\xi) \rightarrow F_{\infty}(x)=\chi_{B_{R_{0}}(0)}(\xi), \quad m\left(F_{m}(\xi)\right)^{m} \rightarrow \frac{1}{2 N}\left(R_{0}^{2}-|\xi|^{2}\right)_{+}
$$

The limit on the left is the so-called mesa profile. For further reference, note also that

$$
\int_{r}^{\infty} r F(r) d r=\left(R_{0}^{2}-r^{2}\right)_{+} / 2
$$

All this is to be compared with the calculations for the fractional case, with $0<s<1$, to be developed in the next three sections. 


\section{Review of the fundamental solutions in the fractional case}

We consider next the solution $U_{m}(x, t)$ to the Cauchy problem (1.1)-(1.2) with initial data a Dirac delta, that is

$$
U_{m}(x, 0 ; M)=M \delta(x), \quad M>0, m>1 .
$$

This problem has been studied in [51] where it is proved that for every choice of parameters $s \in$ $(0,1)$ and $m>m_{c}=\max \{(N-2 s) / N, 0\}$ and every $M>0$ the equation admits a unique fundamental solution, which is a nonnegative continuous weak solution for $t>0$ and takes the initial data in the sense of Radon measure, which means that

$$
\lim _{t \rightarrow 0} \int U_{m}(x ; t) \phi(x) d x=M \phi(0)
$$

holds for all $\phi \in C_{b}\left(\mathbb{R}^{N}\right)$. By scaling we can reduce the study to the case $M=1$ through the formula

$$
U_{m}(x, t ; M)=M U_{m}\left(x, M^{m-1} t\right) .
$$

We write in the sequel $U_{m}=U_{m}(x, t ; 1)$. This solution also depends on the parameters $N$ and $s$ but this dependence will be omitted as a rule since it usually plays no part in the arguments. We have the formulas

$$
U_{m}(x, t)=t^{-\alpha} F_{m}(\xi),
$$

where $\xi=x / t^{\beta}$ and now we have the expressions

$$
\alpha=\frac{N}{N(m-1)+2 s}, \quad \beta=\frac{\alpha}{N}=\frac{1}{N(m-1)+2 s} .
$$

Moreover, it has been proved that $F_{m}$ (called the self-similar profile) is a bounded, positive, and Hölder continuous function that goes to zero as $|x|$ goes to infinity. It is also radially symmetric and a monotone decreasing function of $|x|$.

Equation. The self-similar profile $F=F_{m}$ satisfies an elliptic equation

$$
(-\Delta)^{s} F^{m}=\alpha F+\beta y \cdot \nabla F=\beta \nabla \cdot(y F),
$$

so that, putting $s^{\prime}=1-s$ and integrating in $r$, we have

$$
\nabla(-\Delta)^{-s^{\prime}} F^{m}=-\beta y F \text {. }
$$

In radial coordinates this gives

$$
L_{s^{\prime}} F^{m}(r)=\beta \int_{r}^{\infty} s F(s) d s,
$$

where $L_{s^{\prime}}$ the expression of operator $(-\Delta)^{-s^{\prime}}$ acting on radial functions. We recall again that the fundamental profile is a function of several parameters $F(r)=F_{m, s, N, M}(r)$ but only the relevant ones will be mentioned. The scaling group acts on the profiles $F_{M}(r)$ for different masses $M>0$ and indeed we have

$$
F_{M}(r)=\mu^{2 s} F_{1}\left(\mu^{1-m} r\right), \quad M=\mu^{N(m-1)+2 s},
$$

which reduces all calculations to the case $M=1$. Since $N(m-1)+2 s>0$ for $m>m_{c}$ we get $F_{M}(0) \rightarrow \infty$ as $M \rightarrow \infty$. For $m \geqslant 1$ we also have $\lim _{M \rightarrow \infty} F_{M}(r)=\infty$ for all $r>0$. 
Decay at infinity. First estimate. The precise behaviour of the fundamental profiles $F(y)=$ $F_{m, s, N}(y)$ as $y \rightarrow \infty$ is a very important question in the qualitative theory. It is known in the linear case $m=1$, since then $F$ is given by a linear kernel $K$ that decays like $|y|^{-(N+2 s)}$, [13]. The exact rate of decay for $m \neq 1$ is a nontrivial issue that has been carefully examined by the author in [51] where it is proved that as $r \rightarrow \infty$ we have (at least for $m \geqslant 1$ )

$$
\lim _{r \rightarrow \infty} r^{N+2 s} F_{m, s, N}(r)=c(m, s, N)>0,
$$

but this estimate is not known to be uniform in $m$ for large $m$. A less precise but uniform estimate is obtained by using the fact that $F$ is monotone as a function of $r$ and also integrable in $\mathbb{R}^{N}$. Since we have the mass estimate $\int_{0}^{\infty} F_{m}(r) r^{N-1} d r=M / N \omega_{N}$ and we know that $F_{m}$ is monotone decreasing, we conclude that

$$
0 \leqslant F_{m}(r) \leqslant N r^{-N} \int_{0}^{r} F_{m}(s) s^{N-1} d s \leqslant \frac{M}{\omega_{N} r^{N}} .
$$

This is an upper bound that is uniform in $m$. In the sequel we may put $M=1$ without loss of generality in view of the scaling formula (3.4).
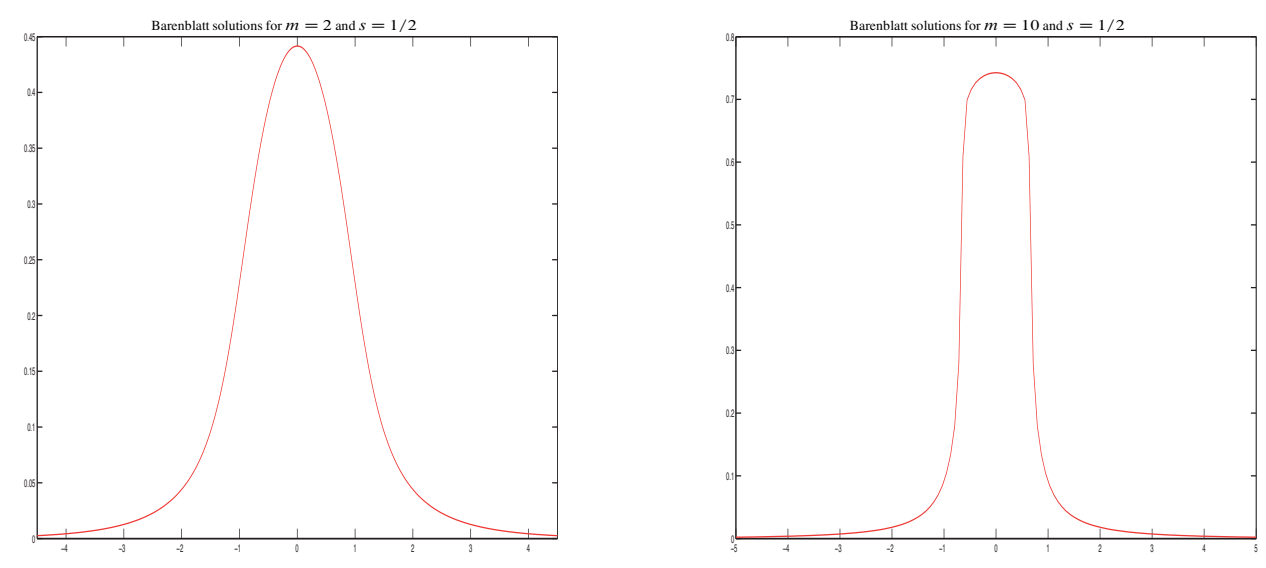

FIG. 1. Transition of the Barenblatt solutions to a mesa with tail in the self-similar profiles. Here, $N=1, s=0.5$ and $m=2$ (left) or $m=10$ (right). (Figures are courtesy of F. del Teso.)

\section{Limit of the fundamental solutions of the FPME}

We are interested in studying the limit of the family of solutions $\left\{U_{m}(r, t) ; m>1\right\}$ as $m \rightarrow \infty$. The parameter $s \in(0,1)$ will be kept fixed. Equivalently, we want to pass to the limit of $F_{m}(r)$ as $m \rightarrow \infty$ for all $r>0$. The observed behaviour is shown in Figure 1 .

\subsection{Limit as $m \rightarrow \infty$ for $N \geqslant 2$}

We begin by assuming that $N \geqslant 2$ since $N=1$ has some subtleties that we treat separately. We introduce the functions $G_{m}(r)=m F_{m}^{m}(r)$, that as $m \rightarrow \infty$ will have a very different behaviour 
compared to $F_{m}$. The idea of using these pairs of functions $\left(F_{m}, G_{m}\right)$ to obtain extra information about the limit comes from the analysis of Section 2 and plays a crucial role in what follows. We recall the notation

$$
W_{m}(x, t)=m U_{m}^{m}(x, t)=t^{-m \alpha} G_{m}\left(x t^{-\beta}\right) .
$$

LEMMA 4.1 Along a subsequence $m_{k} \rightarrow \infty$ we have

(i) $\quad F_{m}(r) \rightarrow F_{\infty}(r)$ in $L^{p}\left(\mathbb{R}^{N}\right)$ for all $1<p<\infty$ and a.e. Moreover, $F_{\infty}$ is a non-increasing radial function, integrable over $\mathbb{R}^{N}$ and $0 \leqslant F_{\infty}(r) \leqslant 1$.

(ii) $\quad G_{m}(r) \rightarrow G_{\infty}(r)$ in $L^{p}\left(\mathbb{R}^{N}\right)$ for $1 \leqslant p<p_{1}=N /(N-2 s)_{+}$and a.e. $G_{\infty}$ is non-increasing radial function, integrable over $\mathbb{R}^{N}$, and $G_{\infty}(r)$ is zero for $r>r_{0}=\omega_{N}^{-1 / N}$.

(iii) If $G_{\infty}(r)>0$ then $F_{\infty}(r)=1$.

Proof. $\quad$ (i) Passing to the limit. Fixing some $r_{1}>0$ we can pass to the limit along a subsequence and we get $F_{m^{\prime}}\left(r_{1}\right) \rightarrow F_{\infty}\left(r_{1}\right)$ as $m^{\prime}=m^{\prime}\left(r_{1}\right) \rightarrow \infty$. Doing the same for a dense countable set $\left\{r_{k}\right\}$ we can get the same result for all $r_{k}$. The limit function $F_{\infty}(r)$ is also nonnegative and monotone and has the same a priori bound, $\omega_{N} r^{N} F_{\infty}(r) \leqslant 1$. It is in principle defined for all $r=r_{k}$, but due to the monotonicity it can be extended to all $r \in(0, \infty)$ by limits from the left for instance, hence it is left-continuous. At all points of continuity the definition coincides with the $\operatorname{limit}_{\lim _{m^{\prime} \rightarrow \infty}} F_{m}(r)$. The rest of the points (jump points) is at most countable. By Egorov's theorem $F_{m} \rightarrow F_{\infty}$ locally in all $L^{p}(0, \infty), 1 \leqslant p<\infty$, with strong convergence. At this stage it might happen that the limit $F_{\infty}$ will contain a Dirac delta at $r=0$, but this will be excluded later by establishing convergence in some $L^{p}$ spaces, $p>1$.

(ii) Uniform estimates for $G_{m}(r)$. If we put $r_{0}=\omega_{N}^{-1 / N}$ and use estimate (3.6) with $M=1$, we have for all $r>r_{0}$

$$
G_{m}(r) \leqslant m\left(\omega_{N} r^{N}\right)^{-m}=m\left(r_{0} / r\right)^{N m}
$$

which tends very fast to zero, uniformly on any interval of the form $\left[r_{0}+\varepsilon, \infty\right)$, so that

$$
\lim _{m \rightarrow \infty} G_{m}(r)=0, \quad r>r_{0},
$$

uniformly for $r \geqslant r_{0}+\varepsilon$. Therefore, $G_{\infty}$ has compact support. Obtaining a uniform estimate on $G_{m}(r)$ for $r<r_{0}$ is more delicate and we need a different kind of argument. Taking any $r_{1} \in\left(0, r_{0}\right)$, by the monotonicity of $G_{m}$ w.r.t. $r$ we have $G_{m}(r) \geqslant G_{m}\left(r_{1}\right)$ for $0<r<r_{1}$, so that

$$
\int_{|x| \leqslant r_{1}} G_{m}(r) d x \geqslant \omega_{N} G_{m}\left(r_{1}\right) r_{1}^{N} .
$$

Using the formula for the Riesz kernel, we conclude that for all $r \geqslant 2 r_{1}$ we have a constant $c_{1}$ (not depending on $m$ ) such that

$$
(-\Delta)^{-s^{\prime}} G_{m}(x) \geqslant c_{1} G_{m}\left(r_{1}\right) r_{1}^{N} r^{-N+2 s^{\prime}}, \quad r=|x| .
$$

But on the other hand, we know the uniform bound $F_{m}(r) \leqslant c_{2} r^{-N}$ for all large $r$, therefore

$$
\int_{r}^{\infty} s F_{m}(s) d s \leqslant c_{3} r^{-N+2} \text { for all } r>0 .
$$

Note that for $N=2$ the last integral is just the mass in the exterior of a ball, and it is bounded above by 1. Comparing both formulas via equation (3.3) and using the fact that $m \beta \rightarrow 1 / N$ we get

$$
G_{m}\left(r_{1}\right) r_{1}^{N} \leqslant c_{4} r^{2 s}, \quad r \geqslant 2 r_{1}
$$


In conclusion, after fixing $r=2 r_{1}$ we get the second uniform estimate

$$
G_{m}\left(r_{1}\right) \leqslant c_{5} r_{1}^{-(N-2 s)}
$$

and $c_{1}, \ldots, c_{5}$ do not depend on $m$.

The two uniform estimates that we have just obtained for $G_{m}$ mean that $G_{m}(x)$ is uniformly integrable for all large $m$ :

$$
\int_{\mathbb{R}^{N}} G_{m}(x) d x \leqslant C<\infty \quad \text { for all } m \geqslant 1
$$

and $C$ does not depend on $m$. In fact, when $m$ is large $G_{m}(x)$ is uniformly bounded in all the spaces $L^{p}\left(\mathbb{R}^{N}\right)$ if $1 \leqslant p<p_{1}=N /(N-2 s)$. The details for the previous estimate are a bit different in dimension $N=1$, see below.

(iii) Limit of $G_{m}(r)=m F_{m}^{m}(r)$ as $m \rightarrow \infty$. We can now apply the same argument used for $F_{m}$ and prove that $G_{m}(r)$ converges to some $G_{\infty}(r)$ along some subsequences not only a.e. but also in all $L^{p}\left(\mathbb{R}^{N} \backslash B_{\varepsilon}(0)\right), 1 \leqslant p<p_{1}$, with strong convergence, and

$$
G_{\infty}(x) \leqslant H(|x|)=c_{5}|x|^{-(N-2 s)} \chi_{B_{r_{0}}(0)}(x) .
$$

This convergence eliminates the possibility of having a Dirac delta at $r=0$ in the limit. The same happens with $F_{\infty}(r)$.

(iv) More on the limit of $F_{m}(r)$ as $m \rightarrow \infty$. The previous results for $G_{m}(r)$ immediately imply that $F_{\infty}(r) \leqslant 1$ for all $r>0$. Actually, for all $r>0$

$$
F_{m}^{m}(r) \rightarrow 0
$$

along subsequences $m^{\prime} \rightarrow \infty$. Indeed, since the estimate for $G_{m}$ is uniform, we can use the whole sequence $m \rightarrow \infty$ and we do not have to take subsequences. This also means that for every $r_{1}>0$ there exists $m_{1}\left(r_{1}\right)$ large enough such that $F_{m}^{m}(r)<1 / 2$ for $r \geqslant r_{1}$ and $m \geqslant m_{1}$, and this means that $F_{m}(r) \leqslant 1$ in the same domain. On the other hand, near the origin $F_{m}(x) \in L^{m}$ with uniform norm, hence the limit $F_{m} \rightarrow F_{\infty}$ takes place in $L^{p}$ for all $p<\infty$.

Remark. It is easy to see that $G_{\infty}>0$ implies $F_{\infty}=1$. It will important to know that $F_{\infty}=1$ iff $G_{\infty}>0$, but this will proved as part of the identification of the limit in Section 5.

Next, we establish that mass is conserved in the limit by estimating the amount of mass on the far field (in other words, in the tails).

LEMMA $4.2 F_{m} \rightarrow F_{\infty}$ in $L^{1}\left(\mathbb{R}^{N}\right)$ and $\int F_{\infty}(|x|) d x=1$.

Proof. (i) We take a nonnegative non-increasing cutoff function $\zeta(x)$ such that $\zeta(x)=1$ for $0<$ $|x|<1, \zeta(x)=0$ for $|x|>2$ and define $\zeta_{R}(x)=\zeta(x / R)$. We also put $\psi_{R}(x)=1-\zeta_{R}(x)$. We calculate the change in the weighted mass of the fundamental solutions $U_{m}$ between $t=0$ and $t=T$ for all large $m$. We take the fundamental solution with mass $M=1$ without loss 
of generality. We have

$$
\begin{aligned}
1-\int_{\mathbb{R}^{N}} U_{m}(x, T) \zeta_{R}(x) d x & =\int_{\mathbb{R}^{N}} U_{m}(x, T) \psi_{R}(x) d x-\int_{\mathbb{R}^{N}} U_{m}(x, 0) \psi_{R}(x) d x \\
& =\int_{0}^{T} \int_{\mathbb{R}^{N}} \partial_{t} U_{m}(x, t) \psi_{R}(x) d x d t \\
& =-\int_{0}^{T} \int_{\mathbb{R}^{N}}(-\Delta)^{S} U_{m}^{m}(x, t) \psi_{R}(x) d x d t \\
& =-\int_{0}^{T} \int_{\mathbb{R}^{N}} U_{m}^{m}(x, t)\left((-\Delta)^{S} \psi_{R}\right)(x) d x d t \\
& =-\left.\int_{0}^{T} t^{N \beta-m \alpha} \int_{\mathbb{R}^{N}} F_{m}^{m}(y)\left((-\Delta)^{S} \psi_{R}\right)\right|_{x=y t^{\beta}} d y d t=(*)
\end{aligned}
$$

We have introduced the self-similar space variable $y=x t^{-\beta}$. Of course, $U_{m}^{m}$ means $\left(U_{m}\right)^{m}$ and likewise for the notation $F_{m}^{m}$. Due to the scaling property of $\psi$

$$
\left((-\Delta)^{s} \psi_{R}\right)(y)=R^{-2 s}\left((-\Delta)^{s} \psi_{1}\right)(y / R)
$$

and we also know that $(-\Delta)^{s} \psi_{1}$ bounded in all $L^{p}$ spaces $1 \leqslant p \leqslant \infty$.

(ii) We now go back to $(*)$ to point out the estimate

$$
\begin{aligned}
\mid \int_{0}^{T} t^{N \beta-m \alpha} \int_{\mathbb{R}^{N}} F_{m}^{m}(y) & (-\Delta)^{s} \psi_{R}(y) d y d t \mid \\
& \leqslant R^{-2 s} \mid \int_{0}^{T} \frac{t^{N \beta-m \alpha}}{m}\left\|G_{m}\right\|_{1}\left\|(-\Delta)^{s} \psi_{1}(y / R)\right\|_{\infty} d t \\
& =\frac{C\left\|G_{m}\right\|_{1}}{m(1+N \beta-m \alpha)} R^{-2 s} T^{1+N \beta-m \alpha} .
\end{aligned}
$$

Recall that $\left\|G_{m}\right\|_{1}$ is uniformly bounded by Lemma 4.1. After observing that $m(1+N \beta-$ $m \alpha)=2 \operatorname{sm} \beta \sim 2 s / N$ as $m \rightarrow \infty$, we get

$$
\lim _{m \rightarrow \infty} \frac{T^{1+N \beta-m \alpha}}{m(1+N \beta-m \alpha)} \rightarrow \frac{N}{2 s}
$$

so that, putting $T=1$,

$$
\left|\int_{0}^{1} t^{N \beta-m \alpha} \int_{\mathbb{R}^{N}} F_{m}^{m}(y)(-\Delta)^{s} \psi_{R}\left(y t^{\beta}\right) d y d t\right| \leqslant C R^{-2 s}
$$

for all large $m$ and $R$, where $C$ does not depend on $R$ or $m$. Going back to the beginning of the calculation, it follows that

$$
\left|1-\int_{\mathbb{R}^{N}} U_{m}(x, 1) \zeta_{R}(x) d x\right| \leqslant C R^{-2 s} .
$$


From this we conclude the convergence of $F_{m}$ to $F_{\infty}$ in $L^{1}\left(\mathbb{R}^{N}\right)$ and also that

$$
\int_{\mathbb{R}^{N}} F_{\infty}(x) \zeta_{R}(x) d x=\int_{\mathbb{R}^{N}} U_{\infty}(x, 1) \zeta_{R}(x) d x=1 .
$$

Let us now perform a further analysis of the form of $F_{\infty}$.

Lemma 4.3 There exists $R>0, R<r_{0}$, such that $F_{\infty}(r)=1$ for $r<R$ and $0<F_{\infty}(r)<1$ for $r>R$. Moreover,

$$
\int_{0}^{\infty} F_{\infty}(r) r^{N-1} d r=\frac{1}{N \omega_{N}} ; \quad F_{\infty}(r) \sim c_{1} r^{-(N+2 s)} \quad \text { as } \quad r \rightarrow \infty .
$$

Moreover, there is an equation relating the profiles

$$
(-\Delta)^{s-1} G_{\infty}(r)=\frac{1}{N} \int_{r}^{\infty} s F_{\infty}(s) d s .
$$

Proof. (i) It follows from the previous lemma that $F_{\infty}$ is monotone, and $\int F_{\infty}(|x|) d x=1$, so that $R \leqslant r_{0}=\omega_{N}^{-1 / N}$, which is finite. $F_{\infty}$ must be less than 1 for $r>R$.

(ii) Next, we establish the equation relating the limit profiles. This is obtained by passage to the limit $m \rightarrow \infty$ in (3.3). The left-hand side is immediate, while for the right-hand side it comes from the Dominated Convergence Theorem if $N \geqslant 3$. For $N=2$ we argue as follows: the expression on the r.h.s. is just the mass of $F_{\infty}$ outside of the ball $B_{r}(0)$. Then we observe that 1.h.s gives a uniform small estimate for the mass of $F_{m}$ and $F_{\infty}(x)$ in the complement of any large ball, and we find a case of tight convergence of probability distributions.

(iii) Let us now use the equation. It is easy to prove that $(-\Delta)^{-s^{\prime}} G_{\infty}(r)$ must be positive for all $r>0$ which means that $\int_{r}^{\infty} s F_{\infty}(s) d s$ cannot be compactly supported, hence neither $F_{\infty}(r)$ is. Actually, since $G_{\infty}(r)$ is compactly supported the decay rate of $(-\Delta)^{-s^{\prime}} G_{\infty}(r)$ is precisely $O\left(r^{-N-2 s^{\prime}}\right)$, and

$$
\int_{r}^{\infty} s F_{\infty}(s) d s=O\left(r^{-N-2 s^{\prime}}\right) .
$$

This and the monotonicity of $F_{\infty}$ imply that the decay rate of $F_{\infty}$ is (in average) $F_{\infty}(r) \sim$ $C r^{-(N+2 s)}$, just as in the finite case $m<\infty$. In any case, $F_{\infty}(r)$ cannot be compactly supported.

(iv) We have to exclude the possibility that $R=0$ in the statement of the lemma. But in that case $G_{\infty}(r)=0$ for all $r>0$. We have to be sure that $G_{\infty}(r)$ is not a Dirac delta, but this has been already excluded by the convergence in some $L^{p}, p>1$. We can exclude it here in a different way: using the equation we would conclude that then

$$
\int_{r}^{\infty} s F_{\infty}(s) d s=C r^{-\left(N-2 s^{\prime}\right)},
$$

which means $F_{\infty}(r)=c_{1} r^{-(N+2 s)}$ for all $r$. This contradicts the previous conclusion $F_{\infty}(r) \leqslant 1$. Summing up, $R=0$ would mean $G_{\infty}(r) \equiv 0$, and using (4.6) this would imply that $F_{\infty} \equiv 0$, which goes against the conservation of mass. Therefore $0<R<r_{0}<\infty$. 


\subsection{Limit of the fundamental solutions in $1 D$}

Let us examine the proofs of this section when $N=1$. Some problems arise: thus, when $s \leqslant 1 / 2$ so that $s^{\prime} \geqslant 1 / 2,2 s^{\prime}-N \geqslant 0$, and the argument of the Lemma 4.1 has a problem at the start since the kernel involves a positive power of $|x-y|$ (or a logarithm for $s=1 / 2$ ). Moreover, even if equation (3.3) holds for every finite $m>1$, the estimate on the asymptotic behaviour that ensures that the r.h.s. integral is finite is not uniform in $m$, and the uniform estimate we have $\int_{\mathbb{R}} F_{m}(x) d x=1$ is not sufficient. The problem is however limited to justifying that the same conclusions hold, and this is what we do next.

(i) Our approach consists in taking the differentiated version (3.2), i.e.,

$$
-\partial_{x}\left(-\partial_{x x}\right)^{-s^{\prime}} G_{m}(r)=m \beta r F_{m}(r) .
$$

Using the integral kernel for $\left(-\partial_{x x}\right)^{-s^{\prime}}$ and differentiating we get a representation for the operator $A=-\partial_{x}\left(-\partial_{x x}\right)^{-s^{\prime}}$ (at least for smooth $f$ )

$$
\begin{gathered}
A f(x)=c \int_{-\infty}^{x} \frac{f(y)}{(x-y)^{2-2 s^{\prime}}} d y-c \int_{x}^{\infty} \frac{f(y)}{(y-x)^{2-2 s^{\prime}}} d y \\
\quad=c \int_{-\infty}^{x} \frac{f(y)-f(2 x-y)}{(x-y)^{2-2 s^{\prime}}} d y
\end{gathered}
$$

where $c=c(s)>0$. In this formula we have to be careful with the cancellations. By the monotonicity of $G_{m}$ we have nonnegative integrand for $A G_{m}(x)$ if $x>0$. Then,

$$
A G_{m}(x) \geqslant c \int_{-r_{0}}^{r_{0}} \frac{G_{m}(y)-G_{m}(2 x-y)}{(x-y)^{2 s}} d y=c \int_{-r_{0}}^{r_{0}} \frac{G_{m}(y)}{(x-y)^{2 s}} d y-c \int_{2 x-r_{0}}^{2 x+r_{0}} \frac{G_{m}(y)}{(y-x)^{2 s}} d y .
$$

Due to the high decay rate of $G_{m}(x)$ for $x>2 r_{0}$ if $m$ is very large, the last integral is very small, uniformly in $m \gg 1$ and $x>2 r_{0}$. Hence, we conclude in the same spirit of the previous calculation for $N \geqslant 2$ that for all $x \geqslant 2 r_{0}$ we have a constant $c_{1}$ (not depending on $m$ ) such that

$$
\left|A G_{m}(x)\right| \geqslant c_{1}\left(\int_{-r_{0}}^{r_{0}} G_{m}(x) d x\right) r^{-2+2 s^{\prime}}-\varepsilon, \quad r=|x| .
$$

Since on the other hand, $2 x F_{m}(x) \leqslant 1$ (by the integrability and monotonicity of $F_{m}$ ) we get the estimate

$$
\left\|G_{m}\right\|_{L^{1}\left(B_{r_{0}}\right)} \leqslant c_{2} r^{1+2 s} F_{m}(r)+\varepsilon r^{2 s} \leqslant c_{3} r^{2 s}, \quad r \geqslant 2 r_{0} .
$$

Fix now $r=2 r_{0}$ to get a uniform estimate and in the limit the conclusion that $G_{\infty} \in L^{1}\left(B_{r_{0}}\right)$, hence $G_{\infty} \in L^{1}(\mathbb{R})$.

(ii) In order to improve that estimate we have two cases, depending on $s$ being small or not. Thus, when $1 / 2<s<1$ operator $A$ has symbol $-i \xi /|\xi|^{-2 s^{\prime}}=-i|\xi|^{2 s-1} \operatorname{sign}(\xi)$, so the fact that $A G_{m}$ is bounded and that $G_{m} \in L^{1}(\mathbb{R})$ (uniformly in $m$ ) implies that $A G_{m}$ is bounded in some fractional Sobolev space and this implies that $G_{m}$ is uniformly in some Hölder space, and so it $G_{\infty}$. Note that we only need the result in a ball around the origin. In case $s=1 / 2$, then $A$ is a Hardy transform (but for a constant) and we conclude that $G_{m}$ is in $L^{p}$ for every $p$, uniformly in $m$, and so is $G_{\infty}$. 


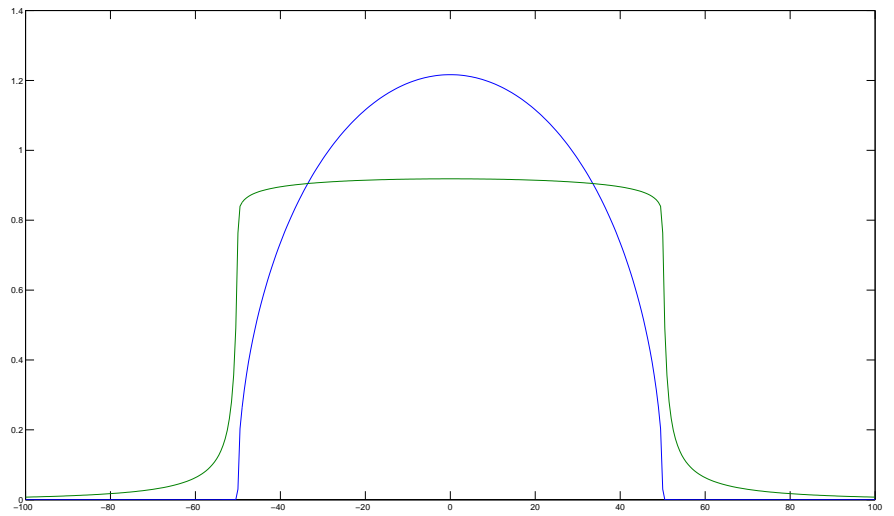

FIG. 2. Formation of the mesa shape in $F_{m}$ and the concave function $G_{m}$ for $m=20$. The scale on the vertical axis is real for $F_{m}$.

When $0<s<1 / 2$ we expect an estimate of the possible singularity at $x=0$ like (4.4). We argue as follows: we take a small $x>0$ and look at the kernel expression for $A G_{m}$ as before, but now we select the interval $x / 3<y<2 x / 3$ to get

$$
A G_{m}(x) \geqslant c \int_{x / 3}^{2 x / 3} \frac{G_{m}(y)-G_{m}(2 x-y)}{(x-y)^{2 s}} d y \geqslant c\left(G_{m}(2 x / 3)-G_{m}(4 x / 3)\right) x^{1-2 s}
$$

so that, using the equation $\left|A G_{m}(x)\right| \leqslant c x F_{m}(x) \leqslant C_{1}$, we get

$$
\left(G_{m}(2 x / 3)-G_{m}(4 x / 3)\right) x^{1-2 s} \leqslant C_{2} .
$$

After applying this in a dyadic sequence $x_{k}=x_{0} 2^{-k}$ and putting $z_{k}=2 x_{k} / 3$ we get $G_{m}\left(z_{k}\right) \leqslant$ $C_{3} z_{k}^{-(1-2 s)}$, as we wanted to prove. We sum up the results.

LEMMA 4.4 The statement of Lemma 4.1 is true without change for $N=1$ if $2 s<1$. When $s=$ $1 / 2$ there is no restriction on $p$ in the convergence of $G_{m} \rightarrow G_{\infty}$, when $s>1 / 2$ the convergence is uniform (and in some Hölder space). The rest of the statements hold.

Once this is established, the rest of the analysis of $G_{m}$ and $F_{m}$ of the section holds too with small changes that are not difficult. In Figure 2 we represent the functions $F_{m}$ and $G_{m}$ for large $m=20$, already showing approximation to their limit shapes.

\section{Characterization of the limit - Obstacle problem}

The support of $G_{\infty}$ is some interval $\left[0, R_{1}\right]$ with $R_{1} \leqslant R$. We have also concluded that $G_{\infty}$ belongs to some $L^{p}$ space with $p>1$. Let us now introduce the function

$$
P_{\infty}(r)=\frac{1}{N} \int_{r}^{\infty} s F_{\infty}(s) d s
$$


and let us also write without fear of confusion $P_{\infty}(x)=P_{\infty}(r)$. Due to the form of $F_{\infty}$ and the fact that $P(0)=C$ is finite, we conclude that

$$
P_{\infty}(r)=C-r^{2} /(2 N) \quad \text { for } 0<r<R, \quad P_{\infty}(r) \geqslant C-r^{2} /(2 N) \quad \forall r>0 .
$$

Hence, we know the exact shape of $P_{\infty}(r)$ near $r=0$ up to a constant. Moreover, for all $r>0$ it is always above the obstacle $\Phi(r)=C-r^{2} /(2 N)$. Here $C$ is a positive constant that may depend on the subsequence $m_{k}$ we have taken, but we will eliminate this restriction below by uniqueness.

We also know that $(-\Delta)^{s^{\prime}} P_{\infty}(x)=G_{\infty}(x)$, at least in a weak sense, see equation (4.6). We can now present the Obstacle Problem we have announced in Theorem 1.2, that we recall here for convenience:

Problem. Given the obstacle function $\Phi(r):=C-\left(r^{2} / 2 N\right), C>0$, to determine two radial nonnegative functions $G$ and $P$ such that

$$
\begin{aligned}
& G(r)(P(r)-\Phi(r))=0 \quad \text { i. e., either } G=0 \text { or } P \text { equals the obstacle; } \\
& P(r) \geqslant C-r^{2} /(2 N) \text { everyshere, } \quad P(r) \rightarrow 0 \quad \text { as } r \rightarrow \infty \\
& (-\Delta)^{s^{\prime}} P(|x|)=G(|x|) \quad \text { in } \quad \mathbb{R}^{N} .
\end{aligned}
$$

In particular, $(-\Delta)^{s^{\prime}} P(r)=0$ on the non-contact set where $P(r)>C-r^{2} /(2 N)$.

The reader could be surprised to find that the obstacle problem is formulated in terms of the two variables $G$ and $P$, and not the original profile $F$. This is quite remarkable in our opinion, even if $F$ is easily obtained from $P$.

Regarding the solution of this problem, for any given $C>0$ the Caffarelli-Silvestre theory [37], [16], [1], says that it has a solution and it is unique. Both $G$ and $P$ are monotone nonincreasing in $r$. The estimated regularity is $C^{1, s^{\prime}}\left(\mathbb{R}^{N}\right)$ for $P(x)$ and $C^{1-s^{\prime}}=C^{s}\left(\mathbb{R}^{N}\right)$ for $G(x)$.

Scaling and uniqueness. Since our functions $P_{\infty}(r)$ and $G_{\infty}(r)$ satisfy the assumptions for some $C>0$, they coincide with the unique solution of the obstacle problem, and the stated regularity applies to them, in particular to $P_{\infty}$. Then, $r F_{\infty}(r)=N P_{\infty}^{\prime}(r) \in C^{s^{\prime}}$, hence away from zero $F_{\infty}(r) \in C^{s^{\prime}}$, while near zero $F_{\infty}$ is constant equal to 1 .

Since the solution of the Obstacle Problem depends on the constant $C$ there is in principle an infinite family of possible solutions. The uniqueness of $C$ depends on the mass conservation law that fixes $\int F_{\infty}(x) d x=1$. To find the appropriate $C$ we argue by scaling in this way: when we pass from our normalized mass 1 to mass $M>0$ we have a whole sequence of solutions of the limit problem given by the formulas

$$
F_{M, \infty}(r)=F_{\infty}(r / M)
$$

(this is a simple scaling formula that is to be compared with the scaling formula (3.4) for finite $m$ by putting $\left.\mu^{m-1}=R\right)$. Then, $P_{M, \infty}(r)=M^{2} P_{\infty}(r / M)$. Therefore, we get in this way all the possible constants $C_{M}=M^{2} C_{1}$, a one-to-one correspondence between mass $M$ and constant $C_{M}$.

We conclude from this analysis that there is a unique $C=C_{1}$ for which the mass of $F=F_{\infty}$ is 1 , and this ends the proof of uniqueness. Uniqueness implies that not only a subsequence $m^{\prime}=m_{k}$ converges but the whole sequence $m \rightarrow \infty$ does.

Moreover, we see that the sequence of continuous and monotone decreasing functions $F_{m^{\prime}}(r)$ converges to a continuous and monotone decreasing function $F_{\infty}(r)$, hence this convergence is locally uniform. 


\subsection{Connection with the CV-fractional diffusion model and explicit formulas}

The obstacle problem we have just discussed was derived by Caffarelli and the author [18] in the study of self-similarity for the fractional diffusion model

$$
u_{t}=\nabla \cdot\left(u \nabla(-\Delta)^{-\sigma / 2} u\right) .
$$

Indeed, the existence and uniqueness of the self-similar solution of the form $U(x, t)=$ $t^{-\alpha_{1}} F_{1}\left(x t^{-\beta_{1}}\right)$, was reduced to find a solution $F_{1}(y)$ of that obstacle problem, and this is done via the results of [16]. The similarity exponents are $\beta_{1}=1 /(N+2-\sigma)$ and $\alpha_{1}=N \beta_{1}$ (they play no role in what follows). ${ }^{*}$ The existence and basic properties for this equation were established in [17].

It is quite interesting that Biler et al. gave in [10-12] an explicit formula for the self-similar solution of the evolution equation, that gives for the profile function the expression

$$
F_{1}(x)=\left(A-B|x|^{2}\right)_{+}^{1-(\sigma / 2)}
$$

with $A$ and $B$ suitable positive constants. The proof of this formula is based on the remarkable explicit formula

$$
(-\Delta)^{\alpha / 2}\left(1-|y|^{2}\right)_{+}^{\alpha / 2}=K(\alpha, N)>0 \text { for }|y|<1 .
$$

due to Blumental-Getoor's [13] and valid for $0 \leqslant \alpha \leqslant 2$.

In order to apply this explicit result to our problem, we recall that $(-\Delta)^{s^{\prime}} P_{\infty}=G_{\infty}(x)$. As mentioned above, the pressure is given by $P_{\infty}(x)=\left(R^{2}-|x|^{2}\right) /(2 N)$ in the so-called coincidence set $\{P=\Phi\}$ which is the ball of radius $R$, so that $-\Delta P_{\infty}=1$. Hence,

$$
(-\Delta)^{s} G_{\infty}(x)=(-\Delta)^{s}(-\Delta)^{s^{\prime}} P_{\infty}=1 \text {. }
$$

Therefore, we use Blumental-Getoor's formula with $\alpha=2 s$. We have

$$
(-\Delta)^{s} A\left(R^{2}-|x|^{2}\right)_{+}^{s}=A R^{2 s}(-\Delta)^{s}\left(1-|x / R|^{2}\right)_{+}^{s}=\left.A(-\Delta)^{s}\left(1-|y|^{2}\right)_{+}\right|_{y=x / R}=A K
$$

for $|x| \leqslant R$. In this way we get the explicit solution

$$
G_{\infty}(x)=\frac{1}{K}\left(R^{2}-|x|^{2}\right)_{+}^{s},
$$

As said before, $R$ is calculated from the mass of $F_{\infty}$, but the calculation must involve the outer values of $F_{\infty}(r)=N P_{\infty}^{\prime}(r) / r$. There are formulas for that expression but we do not need to go into such smaller details.

Since we have an explicit expression for $G_{\infty}$, using formula (4.6) and differentiating w.r.t. $r=$ $|x|$ we get

$$
F_{\infty}(r)=-\frac{N}{r K} \partial_{r}(-\Delta)^{s-1}\left(R^{2}-|x|^{2}\right)_{+}^{s} .
$$

Using now the integral formula for the inverse Laplacian operator, we have

COROLlARY 5.1 The precise asymptotic behavior for $F_{\infty}$ is $\lim _{r \rightarrow \infty} r^{N+2 s} F_{\infty}(r)=c>0$

This was the only assertion pending to be proved to complete Theorem 1.2.

$\$$ The equation is written in [18] in terms of $s=\sigma / 2$ but this use of $s$ conflicts with the exponent $s$ used in this paper for the algebraic formulas examined in this subsection, hence the change of notation. 
Remarks.

(1) The explicit formulas show that the positivity set of $G_{\infty}$ is the same as the interior of the ball where $F_{\infty}=1$ (i.e., the flat set of $F_{\infty}$ ).

(2) In the comparison with equation (1.5), we see that $\sigma=2-2 s$ (our $s$ ) and $F_{1}$ is replaced by $G_{\infty}$

(3) The connection between the two equations that is described here has been extended to a more general correspondence in work with Stan and del Teso, [39]. Indeed, we find an exact correspondence between the fundamental solutions of equation (1.1) with finite exponent $m>1$, and the porous medium equation with nonlocal pressure, $u_{t}=\nabla \cdot\left(u^{m^{\prime}-1} \nabla(-\Delta)^{-s^{\prime}} u\right)$, when $m^{\prime}=(2 m-1) / m, s^{\prime}=1-s$. Note that $m \in[1, \infty)$ gets transformed into $m^{\prime} \in[1,2)$, and the case we have discussed before is the limit $m=\infty, m^{\prime}=2$.

(4) It is interesting to compare the results of this section for $0<s<1$ with the explicit computations performed for the standard PME in Section 2. The limit $s \rightarrow 1$ of the present results gives correct answers. The main qualitative difference is that $F_{\infty}$ is compactly supported for $s=1$, while it is not for $s<1$. Actually, the obstacle problem simplifies drastically when $s \rightarrow 1$. Then, $s^{\prime}=0$, so that $P=G$ and the alternative $G(P-\Phi)=0$ becomes $G=\Phi_{+}$, which is is a parabola continued by zero, as we have calculated in (2.6).

(5) The difference between the initial data of the limit process, which is $M \delta(x)$, and the value of the limit $U_{m}(x, t)$ for $t>0$, which is $F_{\infty}(x)$ is very striking, but is known in the theory of the standard mesa problems and explained as a consequence of the singular character of the limit. It takes the form of an initial discontinuity or initial layer. In the next section we will expand on this issue for our equation. References to occurrences of the phenomenon for other equations or problems will be given in the final Section.

\section{The limit for more general solutions}

We now consider the passage to the limit $m \rightarrow \infty$ for a general initial datum given by a function $u_{0} \geqslant 0$ that is bounded and integrable, $u_{0} \in L^{1}\left(\mathbb{R}^{N}\right) \cap L^{\infty}\left(\mathbb{R}^{N}\right)$. For convenience we sometimes assume it to be compactly supported too. We denote by $u_{m}(x, t)$ the solution of the Cauchy problem with exponent $m>1$ and fixed data $u_{0}$. The existence, uniqueness and properties of these solutions is studied in [30, 31]. Figures 3 and 4 below illustrate the behaviour that we expect. Note that $m=10$ produces graphs similar to $m=\infty$.

\subsection{Main facts}

We want to pass to the limit in the family $\left\{u_{m}(x, t)\right\}_{m>1}$. The existence and properties of the limit will depend on various a priori estimates which are uniform in $m$, and will happen up to subsequences. We have to justify the type of convergence and this is what we do next.

$L^{p}$ bounds. First of all, it is known that for every $m>1$ and every $p \geqslant 1$ we have the estimate

$$
\left\|u_{m}(\cdot, t)\right\|_{p} \leqslant\left\|u_{0}\right\|_{p} .
$$

This first uniform bound allows us to pass to the limit weakly-* in $L^{\infty}\left(Q_{T}\right)$ and weakly in $L^{p}\left(\mathbb{R}^{N}\right)$ for all $p<\infty$, along a subsequence that we denote by $m^{\prime}$, to obtain a limit function $u_{\infty}(x, t) \in$ $L^{\infty}\left(Q_{T}\right) \cap L^{\infty}\left(0, T: L^{1}\left(\mathbb{R}^{N}\right)\right)$. 

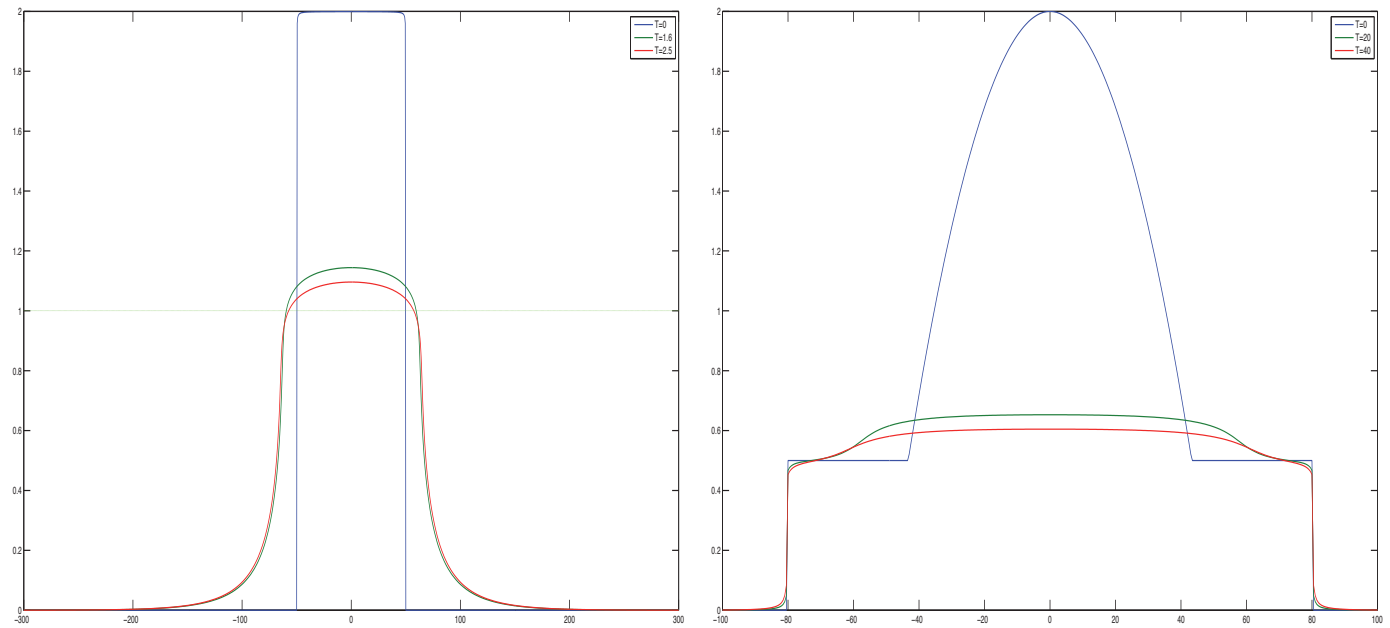

FIG. 3. Collapse of solutions to the level $u=1$. On the right, effect of a lateral step with height less than 1 . In both figures $m=10$.
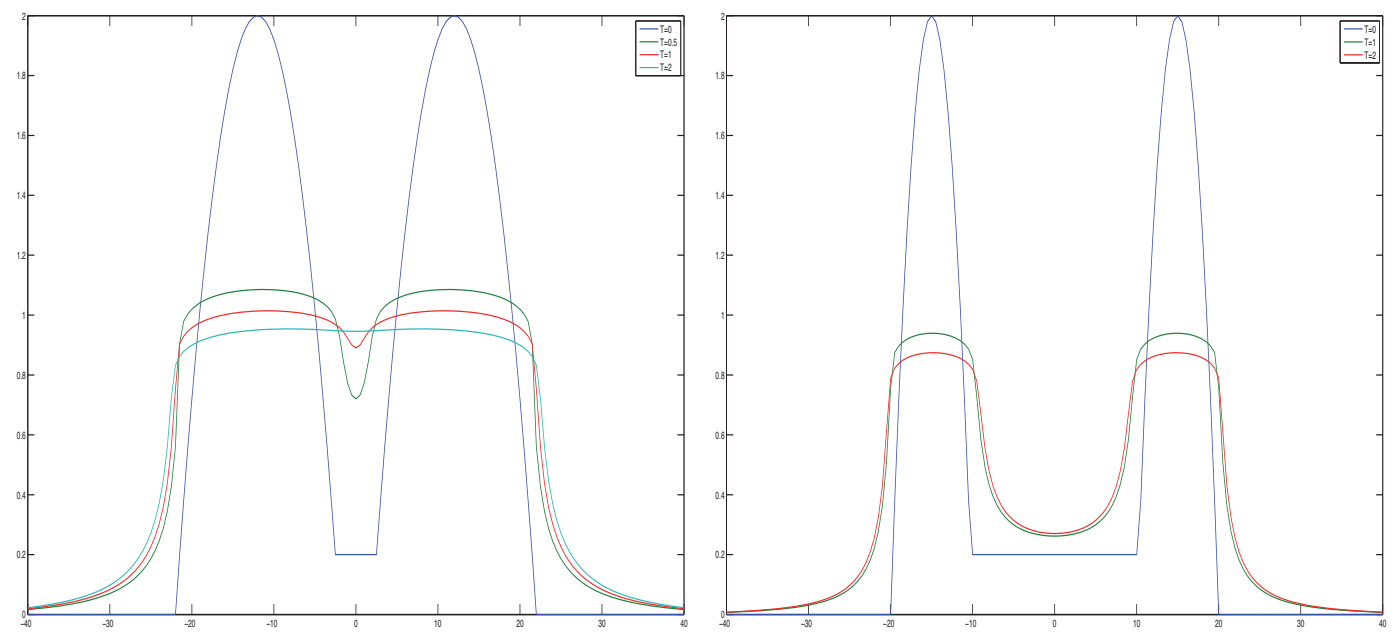

FIG. 4. Interaction of two humps at several times. Here $m=10$

Contractivity. We have a stronger property in the $L^{1}\left(\mathbb{R}^{N}\right)$ norm: for two solutions $u_{m}$ and $\widehat{u}_{m}$ with initial data $u_{0}$ and $\widehat{u}_{0} \in L^{1}\left(\mathbb{R}^{N}\right)$

$$
\left\|u_{m}(t)-\widehat{u}_{m}(t)\right\|_{1} \leqslant\left\|u_{0}-\widehat{u}_{0}\right\|_{1}
$$

and this is valid for all $m \geqslant 1$ and will be also valid in the limit $m \rightarrow \infty$. 
Monotonicity. It is well-known in the FPME theory [5] that for every $m>1$ we have for all nonnegative solutions

$$
\partial_{t} u_{m} \geqslant-\frac{u_{m}}{(m-1) t} .
$$

As a consequence, we have for any limit $u_{\infty}$ of the $u_{m}$

$$
\partial_{t} u_{\infty} \geqslant 0,
$$

which means that under such general initial conditions, every limit function $u_{\infty}(x, t)$ is monotone nondecreasing in time.

Stronger estimate. Stationary limit. Moreover, for every $m>1$ and for all nonnegative solutions with $u_{0} \in L^{1}\left(\mathbb{R}^{N}\right)$ we know that

$$
\left\|\partial_{t} u_{m}\right\|_{1} \leqslant \frac{2\left\|u_{0}\right\|_{1}}{(m-1) t}
$$

As a consequence, we have in the limit $\partial_{t} u=0$ a.e., which means that $u_{\infty}(x, t)$ does not depend on time for $t>0$. In other words, the limit is stationary. This does not mean that necessarily $u_{\infty}(x)=u_{0}(x)$, because the estimate for $u_{m}$ is good only for $t>0$, but is singular near $t=0$. The difference between the initial data of a process and the limit of the values for the solution of the process for $t>0$ is usually labeled in the theory of singular limits as an initial discontinuity or initial layer. Identifying the stationary level $u_{\infty}(x)$ that corresponds to an initial function $u_{0}(x)$ is the main remaining problem of the theory that we are beginning to address here.

Further regularity. Strong limit. Estimate (6.3) implies compactness in time for the sequence $\left\{u_{m}\right.$ : $m>1\}$ that we already knew to be uniformly bounded. Compactness in space depends on the $L^{1}$ contractivity in space.

$$
\int\left|u_{m}(x+h, t)-u_{m}(x, t)\right| d x \leqslant \int\left|u_{0}(x+h)-u_{0}(x)\right| d x
$$

and this quantity goes to 0 as $h \rightarrow 0$. Therefore, the sequence is compact in $L^{1}\left(\mathbb{R}^{N} \times(s, T)\right)$, $0<s<T<\infty$, and the convergence $u_{m} \rightarrow u$ can be assumed from this moment on as being an $L^{1}$ convergence locally in $Q_{T}$, and also a convergence almost everywhere.

The case of simple limit. There is one case in which the identification is simple, and there is no initial discontinuity.

Proposition 6.1 If $0 \leqslant u_{0}(x) \leqslant 1$ then $u_{\infty}(x, t)=u_{0}(x)$.

Proof. Assume first that $u_{0}(x) \leqslant 1-\varepsilon$. In that case we may write the weak solution and easily pass to the limit in the diffusion term to get

$$
\int_{\mathbb{R}^{N}} u_{0}(x) \zeta(x) d x=\int_{\mathbb{R}^{N}} u(x, t) \zeta(x) d x
$$

for every smooth test function $\zeta$, hence, the conclusion. For $u_{0}(x) \leqslant 1$ we may use the $L^{1}$ contraction. We leave these details to the reader. 


\subsection{New upper estimates}

At this moment we have examined two options, the last one where $u_{0}(x)$ is preserved in time, and the other extreme case where $u_{0}(x)$ is a Dirac delta, and then we saw that there is a huge jump from this initial data to the stationary situation $u_{\infty}(x)$ for $t>0$, as we have shown in the first part of this paper. In order to examine some cases of solutions $u$ where $u_{0}$ is an integrable function and $u$ undergoes a jump from $t=0$ to $t=0+$ we will consider initial functions that take values larger than 1 in some nontrivial set. In order to study the limit $m \rightarrow \infty$ we need further estimates.

Uniform boundedness. This is an instance of use of the properties of the limit of the self-similar solutions that we have studied in the previous section.

Proposition 6.2 Suppose that $u_{0} \in L^{1}\left(\mathbb{R}^{N}\right), u_{0}(x) \geqslant 0$. Then for a.e. $x \in \mathbb{R}^{N}$ and $t>0$ we have

$$
u_{\infty}(x, t) \leqslant 1 \text {. }
$$

If moreover $u_{0}$ is bounded and compactly supported, we have

$$
m u_{m}^{m}(x, t) \leqslant C_{1} t^{-m \alpha}
$$

for all $t>0$ and some $C_{1}$ that does not depend on $m$.

Proof. (i) Let us assume that $u_{0}(x) \leqslant C$ and is supported in the ball of radius $R_{0}$. We want to bound above the evolving solutions $u_{m}(x, t)$ by putting on top of them a fundamental solution with some large mass to be adjusted, and also using some small shift in time. The upper bound will be uniform in $m$ for all $m$ large enough. We consider the fundamental solution of unit mass

$$
U_{m}(x, t+\tau)=(t+\tau)^{-\alpha} F_{m}\left(x(t+\tau)^{-\beta}\right),
$$

and then we rescale this solution to mass $M>1$

$$
U_{M, m}(x, t+\tau)=M^{2 s \beta}(t+\tau)^{-\alpha} F_{m}\left(x(t+\tau)^{-\beta} M^{-(m-1) \beta}\right) .
$$

We want to make sure that for some choice of $\tau=\tau_{m}$ and $M$ we have $U_{M, m}\left(R_{0}, 0\right) \geqslant C$, i.e.,

$$
M^{2 s \beta} \tau^{-\alpha} F_{m}\left(R_{0} \tau^{-\beta} M^{-(m-1) \beta}\right) \geqslant C .
$$

Recall that for $m$ very large we have $(m-1) \beta \sim 1 / N$ and $\alpha \sim 0$. Putting $\tau^{\beta}=\lambda$ and recalling that $F_{m} \rightarrow F_{\infty}$ and $m F_{m}^{m}(r) \rightarrow G_{\infty}$ we get sufficient conditions as follows: we first select a radius, say $R_{1} \geqslant R_{0}$ at which $F_{\infty}\left(R_{1}\right) \geqslant c_{1}$, then we put

$$
R_{0} \lambda^{-1} M^{-1 / N} \leqslant R_{1} \quad \text { and need } \quad \lambda^{-N} M^{2 s \beta} \geqslant(C+\varepsilon) / c_{1} .
$$

First, we put $M=\lambda^{-N}$ and the first condition is satisfied. Then, since $2 s \beta \rightarrow 0$, we satisfy the second condition for large $m$ by putting $\lambda=\min \left\{\left(2 C / c_{1}\right)^{-1 / N}, 1 / 2\right\}$. This means that $\tau_{m}=\lambda^{1 / \beta}$ tends to 0 as $m \rightarrow \infty$.

We can now use the comparison result for the FPME. From $u_{m}(x, 0) \leqslant U_{M, m}\left(x, \tau_{m}\right)$ for all $m$ large enough we conclude that for all $x \in \mathbb{R}^{N}$ and $t>0$

$$
u_{m}(x, t) \leqslant U_{M, m}\left(x, t+\tau_{m}\right),
$$

which in the limit gives a limit $u_{\infty}(x, t) \leqslant 1$ for every fixed $t>0$. 
(ii) The calculation also gives an upper bound on the spatial tail of the form $u_{m}(x, t)=$ $O\left(|x|^{-(N+2 s)}\right)$, the same as in the fundamental solution.

(iii) It is not difficult to show that the quantities $w_{m}(x, t)=m\left(u_{m}(x, t)\right)^{m}$ are uniformly bounded and integrable in $x$ by the same comparison trick. Indeed,

$$
m\left\|u_{m}(\cdot, t)\right\|_{\infty}^{m} \leqslant \max _{x} m U_{M, m}^{m}\left(x, t+\tau_{m}\right)=m t^{-m \alpha} F_{M, m}^{m}(0)=G_{M, m}(0) t^{-m \alpha}=C_{1} t^{-m \alpha} .
$$

Recall now that $m \alpha \rightarrow 1$ as $m \rightarrow \infty$. Similar argument for integrability.

(iv) It follows by approximation that estimate (6.4) holds for the limit solution corresponding to any initial data $u_{0} \geqslant 0, u_{0} \in L^{1}\left(\mathbb{R}^{N}\right)$.

Remark. Estimate (6.4) is true for much more general data. We only need a bound of the form

$$
u_{0}(x) \leqslant c+\phi(x), \quad \text { with } c<1 \text { and } \phi(x) \in L^{1}\left(\mathbb{R}^{N}\right) .
$$

We can derive a useful consequence from the two last results and comparison.

Proposition 6.3 We have $u_{\infty}(x, t)=1$ a.e. in the set $\left\{x: u_{0}(x) \geqslant 1\right\}$.

Proof. Define $f=\min \left\{u_{0}, 1\right\}$. The limit of the solutions for the FPME with data $f$ is again $f$, and by comparison $u_{\infty}(x) \geqslant f$. Together with Proposition 6.2 it implies the result.

Therefore, a solution with initial data $u_{0}$ lying somewhere above the line $u=1$ must collapse into a state $u_{\infty}(x) \leqslant 1$. Since the total mass is conserved, see next, this implies that the integral of $u_{\infty}(x)$ on the set $\left\{x: u_{0}(x)<1\right\}$ must be larger than the integral of $u_{0}(x)$ over the same set, hence we must have $u_{\infty}(x)>u_{0}(x)$ in a set of nonzero measure.

Next, we prove the property of mass conservation.

Proposition 6.4 For every $u_{0} \in L^{1}\left(\mathbb{R}^{N}\right), u_{0} \geqslant 0$, and every limit $u_{\infty}(x)$ we have $\int u_{0}(x) d x=$ $\int u_{\infty}(x) d x$.

Proof. We assume first that $u_{0} \in L^{\infty}\left(\mathbb{R}^{N}\right)$. Using a typical cutoff function $\zeta$ and then rescaling it to $\zeta_{R}(x)=\zeta(x / R)$ we have

$$
\begin{aligned}
\mid \int u_{0}(x) \zeta_{R}(x) d x- & \int u_{m}(x, t) \zeta_{R}(x) d x \mid \\
& =\left|\int_{0}^{t} \int u_{m}^{m}(-\Delta)^{s} \zeta_{R} d x d t\right| \\
& \leqslant \int_{0}^{t}\left\|u_{m}(t)\right\|_{\infty}^{(m-1)} d t \int u_{m}(x, t)\left|(-\Delta)^{s} \zeta_{R}\right| d x=\frac{C}{2 s m \beta} R^{-2 s} t^{2 s \beta}
\end{aligned}
$$

where we have used Proposition 6.2 to get the uniform bound

$$
\left\|u_{m}(t)\right\|_{\infty}^{(m-1)} \leqslant C m^{-1+1 / m} t^{-(m-1) N \beta},
$$

and then we have integrated in $t$. Recalling that $m \beta \rightarrow 1 / N$ as $m \rightarrow \infty$, in the limit we get

$$
\left|\int u_{0}(x) \zeta_{R}(x) d x-\int u_{\infty}(x) \zeta_{R}(x) d x\right| \leqslant C_{2} R^{-2 s}
$$


Let now $R \rightarrow \infty$ to conclude the mass conservation rule. For general $u_{0} \in L^{1}\left(\mathbb{R}^{N}\right)$ we use approximation and the property of $L^{1}$ contraction.

\subsection{Control of the initial layer and behaviour at infinity}

At this point we need to introduce a new variable, $h_{m}(x, t)=\int_{0}^{t} u_{m}^{m}(x, s) d s$. In the limit $m \rightarrow \infty$ it will serve as an indicator of the initial collapse that the solution undergoes, and a locator of the resulting 'debris mound', so to say. Integrating in time equation (1.1), we have

$$
(-\Delta)^{s} h_{m}(x, t)=u_{0}(x)-u_{m}(x, t),
$$

which is a basic tool in analyzing the initial layer that appears as $m \rightarrow \infty$. It is instructive to check first the limit in that expression $h_{m}$ in the case of the fundamental solutions. We have for $t=1$

$$
H_{m}(x)=\int_{0}^{1} t^{-N m \beta} F_{m}^{m}\left(r t^{-\beta}\right) d t=\int_{1}^{\infty} \beta^{-1} F_{m}^{m}(r \rho) \rho^{N m-1} \rho^{-1 / \beta} d \rho
$$

where we have put $\rho=t^{-\beta}$ (we use capital letter for the $h_{m}$ function of the fundamental solutions). Therefore, putting $\lambda=N m-\beta^{-1}=N-2 s$

$$
H_{m}(r)=(m \beta)^{-1} \int_{1}^{\infty} G_{m}(r \rho) \rho^{\lambda-1} d \rho
$$

which converges since $G_{m}$ decreases very quickly at infinity if $m$ is large. As $m \rightarrow \infty, H_{m}(r)$ converges to $H_{\infty}(r)=N \int_{1}^{\infty} G_{\infty}(r \rho) \rho^{\lambda-1} d \rho$, which is easy to compute and has compact support.

General solutions. By comparison, the same property of compact support is true for the $h_{\infty}$ corresponding to a bounded and compactly supported initial function $u_{0}$.

In view of the a priori estimates we know that $h_{m}(x, t)$ converges to some $h_{\infty}(x)$ that does depend on $t$ for $t>0$ and we have in the limit

$$
(-\Delta)^{s} h_{\infty}(x)=u_{0}(x)-u_{\infty}(x)
$$

that we call the $h_{\infty}$-equation. Since $h_{\infty}(x)$ has compact support we conclude that $-(-\Delta)^{s} h_{\infty}$ behaves like $c|x|^{-(N+2 s)}, c>0$ as $|x| \rightarrow \infty$. This is precisely the behaviour of $u_{\infty}$ under the assumption that $u_{0}(x)$ has compact support and $h_{\infty}$ is not identically zero. The last situation is implied by the assumption $u_{0}(x) \neq u_{\infty}(x, t)$, and this in turn is true if and only if $u_{0}$ is not equal or less than 1 . Here is the conclusion.

Proposition 6.5 Assume that $u_{0}$ is nonnegative, bounded, compactly supported. If moreover $u_{0}$ is not equal or less than 1 everywhere, then $h_{\infty} \not \equiv 0$ and

$$
u_{\infty}(x) \sim c|x|^{-(N+2 s)} \quad \text { as }|x| \rightarrow \infty .
$$

On the other hand, if $u_{0} \leqslant 1$, then $h_{\infty} \equiv 0$ and $u_{\infty}=u_{0}(x)$, which can have various decay forms as $|x| \rightarrow \infty$. 


\section{Negative result for symmetrization}

Symmetrization techniques are a very popular tool of obtaining a priori estimates for the solutions of different partial differential equations, notably those of elliptic and parabolic type. Symmetrization techniques appear in classical works like [28, 33]. The application of Schwarz symmetrization to obtaining a priori estimates for elliptic problems is already described by Weinberger in [56]. Sharp a priori estimates for the solutions can be derived by using comparison with a model symmetric problem. Pointwise comparison was firmly established in the works of Talenti [41, 42]. For parabolic problems pointwise comparison fails and is replaced by so-called concentration comparison. In the case of the porous medium equation $u_{t}=\Delta u^{m}$ that result was established in [45, 47], and holds for all $m>1$. In order to state the result we want, the following definition is needed:

DEFInition Let $f, g \in L_{\text {loc }}^{1}\left(\mathbb{R}^{N}\right)$ be two radially symmetric functions on $\mathbb{R}^{N}$. We say that $f$ is less concentrated than $g$, and we write $f \prec g$ if for all $R>0$ we get

$$
\int_{B_{R}(0)} f(x) d x \leqslant \int_{B_{R}(0)} g(x) d x .
$$

The partial order relationship $\prec$ is called comparison of mass concentrations. The following result is well-known.

THEOREM 7.1 Let Let $u_{1}, u_{2}$ be nonnegative, weak solutions of the PME $u_{t}=\Delta u^{m}$, posed in $Q=\mathbb{R}^{N} \times(0, \infty)$, with nonnegative initial data $u_{01}, u_{02} \in L^{1}\left(\mathbb{R}^{N}\right)$. Assume that both $u_{02}$ and $u_{01}$ are radially symmetric and $u_{02} \prec u_{01}$. Then, for all $t>0$ we have

$$
u_{2}(\cdot, t) \prec u_{1}(\cdot, t) .
$$

In particular, we have $\left\|u_{2}(\cdot, t)\right\|_{p} \leqslant\left\|u_{1}(\cdot, t)\right\|_{p}$ for every $t>0$ and every $p \in[1, \infty]$.

Recently, such concentration comparison has been extended by the author and Volzone [54] to the fractional Laplacian version $u_{t}+(-\Delta)^{s} u^{m}=0$ for $0<s<1$ and all $m \leqslant 1$, and the authors were surprised to find that the result does not hold for $m>1$. We find here a confirmation for such negative result for the limit case $m=\infty$. As a simple consequence, it cannot hold for large enough $m$ due to the continuity of the limit demonstrated in Section 4.6.

Counterexample. It consists of radial functions. As a first candidate we take an initial function $u_{01}$ such that $0 \leqslant u_{01}(x)=2^{N}$ for all $|x| \leqslant 1$, and $u_{01}(x)=0$ otherwise. As a second candidate, we take $u_{02}(|x|)$ such that $u_{02}(x)=1$ in a ball of radius $R=2$ and $u_{02}(x)=0$ otherwise, so that $\int u_{01}(x) d x=\int u_{02}(x) d x$, and $u_{02} \prec u_{01}$.

However, we know that $u_{\infty, 2}(x)=u_{02}(x)$ is compactly supported, while $u_{\infty, 1}(x)$ decays as $|x| \rightarrow \infty$ like $c|x|^{-(N+2 s)}$, cf. Proposition (6.5). Therefore, it is impossible that $u_{\infty, 2} \prec u_{\infty, 1}$ since both have the same mass (i.e., total space integral). The reader who does not like discontinuous functions will find it easy to adapt the argument and provide an example where $u_{01}$ and $u_{01}$ are continuous and compactly supported functions.

\section{Comments and open problems}

Initial discontinuities happen in other singular problems in diffusion. Let us mention some examples without any pretension to completeness: 
(i) A remarkable case happens in the very fast diffusion equation $u_{t}=\Delta\left(u^{m}\right) / m$; it was proved in [46] that when we consider the initial-value problem in $\mathbb{R}^{N}, N \geqslant 2$, with $m<0$ (singular diffusion), and the initial function is non-negative and integrable, then the candidate solution obtained as limit of regularized approximations vanishes identically for all $t>0$ at all points $x \leqslant \mathbb{R}^{N}$. This strong effect is called instantaneous extinction. The results were then extended by Daskalopoulos and Del Pino [20-22] to classes of non-integrable data, and there are counterparts for $N=1$ with $m \leqslant-1$, see a detailed account in [49].

Added in proof. These results have been now extended to the case of fractional diffusion equation $u_{t}+\left(-\Delta^{s}\left(u^{m}\right) / m=0\right.$ with $m<0,0<s<1$, and the optimal range of $m$ and $s$ for the phenomenon of instantaneous extinction is described in [14] and [53].

(ii) As another type of example, a curious mesa problem was found in logarithmic diffusion with signed data by Rodriguez and the author [35, Section 9], and then an obstacle problem and an initial layer arise, as in our previous study, but of course with different details.

(iii) A third remarkable example appears in the study of the limit as $p \rightarrow \infty$ of the solutions of the $p$-Laplacian equation $u_{t}=\nabla \cdot\left(|\nabla u|^{p-2} \nabla u\right)$. Evans et al. [24] study the Cauchy problem posed in the whole space with initial data $u_{0} \geqslant 0$ and show that, even if the initial datum has a large slope $\left\|\nabla u_{0}\right\|_{\infty}=L>1$, the function obtained in the limit of the $p$-Laplacian problems always satisfies $\|\nabla u(\cdot, t)\|_{\infty} \leqslant 1$. The authors interpret the limit as a crude model for the collapse of a sandpile from an initially unstable configuration. The basic physical assumption is that a sandpile is stable if and only if its slope is everywhere less than or equal to one. Hence, the condition on the initial data implies that the starting profile is unstable. We point out for comparison that the restriction imposed by the limit affects the gradient of the solution, instead of affecting the $L^{\infty}$ norm of the function, as in the PME and the FPME. More information about models with $p \rightarrow \infty$ can be obtained in [2] and [25] for instance. There is also work on so-called doubly nonlinear models, like [7]. On the other hand, an abstract consideration of these singular limits is done in [6] as an homage to the late P. Bénilan.

We will continue in [34] the analysis of the limit $m \rightarrow \infty$ for general data, proving in particular the unique identification of the limit. This is more elaborate work that involves the associated variational inequality problem.

The existence of mesa problems as limits of nonlinear, possibly nonlocal diffusion could be investigated for other interesting models, like the different diffusion plus aggregation models that are being discussed in the literature. The influence of nonlocal diffusion is not well understood for such models.

We do not know what is the correct statement about comparison after symmetrization that will be valid for the solutions of the parabolic problem (1.1)-(1.2) and will be useful in the applications. Any input in this topic will be most welcome.

Acknowledgments Author partially supported by the Spanish project MTM2011-24696. He would like to thank the Isaac Newton Institute for Mathematical Sciences, Cambridge, where this work was completed during the program Free Boundary Problems and Related Topics. The author is grateful to F. del Teso for excellent work to produce the numerical evidence, using the methods of [43] and [44]. He also thanks F. Quirós, B. Volzone, and the anonymous referees for a careful reading of the document and useful suggestions. 


\section{REFERENCES}

1. Athanasopoulos, I., Caffarelli, L. A., \& Salsa, S., The structure of the free boundary for lower dimensional obstacle problems. Amer. J. Math. 130 (2008), 485-498. Zbl1185. 35339 MR2405165

2. Barrett, J. W., \& Prigozhin, L., Bean's critical-state model as the $p \rightarrow \infty$ limit of an evolutionary p-Laplacian equation, Nonlinear Anal., Ser. A: Theory Methods 42 (2000), 977-993. Zbl1170.82436 MR1780448

3. Bénilan, P., Boccardo, L., \& Herrero, M., On the limit of solution of $u_{t}=\Delta u^{m}$ as $m \rightarrow \infty$. In Some Topics in Nonlinear PDEís, Proceedings of the International Conference, Torino, M. Bertsch et al. (eds.), 1989. MR1155452

4. BÉnilan, P., \& CRAndall, M. G., The continuous dependence on $\varphi$ of solutions of $u_{t}-\Delta \varphi(u)=0$. Indiana Univ. Math. J. 30 (1981), 161-177. Zb10482. 35012 MR0604277

5. BÉnilan, P., \& Crandall, M. G., Regularizing effects of homogeneous evolution equations. Contributions to Analysis and Geometry, (suppl. to Amer. Jour. Math.), Johns Hopkins Univ. Press, Baltimore, Md., 1981. Pp. 23-39. Zb10556. 35067 MR0648452

6. BÉnilan,, P., Evans, L. C., \& GARIEPY, R. F., On some singular limits of homogeneous semigroups, J. Evol. Equ. 3 (2003), 203-214. Zbl1035.35026 MR1980973

7. BÉnilan, P., \& IGBIDA, N., La limite de la solution de $u_{t}=\Delta_{p}\left(u^{m}\right)$ lorsque $m \rightarrow \infty$. [The limit of the solution of $u_{t}=\Delta_{p}\left(u^{m}\right)$ as $m \rightarrow \infty$ ]. C. R. Acad. Sci. Paris Sèr. I Math. 321 (1995), 1323-1328. Zb10841.35013 MR1363573

8. BÉNILAN, P., \& IGBIDA, N., Singular limit of the changing sign solutions of the porous medium equation. J. Evol. Equations 3 (2003), 215-224. Zbl1029.35146 MR1980974

9. BÉnilan, P., \& Igbida, N., The Mesa problem for the Neumann boundary value problem. J. Differential Equations 196 (2004), 301-315. Zbl1134.35369 MR2028110

10. Biler, P., Imbert, C., \& KARCH, G., Barenblatt profiles for a nonlocal porous medium equation. $C . R$. Math. Acad. Sci. Paris 349 (2011), 641-645. Zb11221. 35209 MR2817383

11. Biler, P., IMBERT, C., \& KARCH, G., Nonlocal porous medium equation: Barenblatt profiles and other weak solutions, Arch. Ration. Mech. Anal. 215 (2015), 497-529. Zbl1308. 35197 MR3294409

12. Biler, P., Karch, G., \& Monneau, R., Nonlinear diffusion of dislocation density and self-similar solutions. Comm. Math. Phys. 294 (2010), 145-168. Zbl1207. 82049 MR2575479

13. Blumenthal, R. M. \& Getoor, R. K., Some theorems on stable processes. Trans. Amer. Math. Soc. 95 (1960), 263-273. Zbl0107.12401 MR0119247

14. Bonforte, M., Segatti, A., \& VÁzquez, J.L., Non-existence and instantaneous extinction of solutions for singular nonlinear fractional diffusion equations, arXiv:1505.03167 [math.AP].

15. Caffarelli, L., \& Friedman, A., Asymptotic behavior of solutions of $u_{t}=\Delta u^{m}$ as $m \rightarrow \infty$. Indiana Univ. Math. J. 36 (1987), 711-728. Zb10651. 35039 MR0916741

16. Caffarelli, L. A., \& Silvestre, L., An extension problem related to the fractional Laplacian, Comm. Partial Differential Equations 32 (2007), 1245-1260. Zbl1143.26002 MR2354493

17. Caffarelli, L. , \& VÁzquez, J. L., Nonlinear porous medium flow with fractional potential pressure. Arch. Ration. Mech. Anal. 202 (2011), 537-565. Zbl1264.76105 MR2847534

18. Caffarelli, L. , \& VÁzquez, J. L., Asymptotic behaviour of a porous medium equation with fractional diffusion. Discrete Contin. Dyn. Syst. 29 (2011), 1393-1404. Zbl1211. 35043 MR2773189

19. Crandall, M. G., \& Liggett, T. M., Generation of semi-groups of nonlinear transformations on general Banach spaces. Amer. J. Math. 93 (1971), 265-298. Zbl0226. 47038 MR0287357

20. Daskalopoulos, P. , \& Del Pino, M. A., On fast diffusion nonlinear heat equations and a related singular elliptic problem. Indiana Univ. Math. J. 43 (1994), 703-728. Zb10806. 35086 MR1291536

21. Daskalopoulos, P. , \& Del Pino, M. A., On a singular diffusion equation. Comm. Anal. Geom. 3 (1995), 523-542. Zb10851. 35072 MR1371208 
22. DASKalopoulos, P. , \& Del Pino, M. A., On nonlinear parabolic equations of very fast diffusion. Arch. Rational Mech. Anal. 137 (1997), 363-380. Zb10886. 35081 MR1463800

23. Elliott, C. M. , Herrero, M. A., King, J. R., \& Ockendon, J. R., The mesa problem: diffusion patterns for $u_{t}=\nabla \cdot\left(u^{m} \nabla u\right)$ as $m \rightarrow \infty$. IMA J. Appl. Math. 37 (1986), 147-154. Zbl0655.35034 MR0983523

24. Evans, L.C., Feldman, M., \& Gariepy, R F., Fast/slow diffusion and collapsing sandpiles. $J$. Differential Equations 137 (1997), 166-209. Zbl0879. 35019 MR1451539

25. Feldman, M., Variational evolution problems and nonlocal geometric motion. Arch. Ration. Mech. Anal. 146 (1999), 221-274. Zbl0955. 49025 MR1720391

26. Friedman, A., \& Höllig, K., On the mesa problem. J. Math. Anal. Appl. 123 (1987), 564-571. Zb10631.35046 MR0883709

27. Friedman, A., \& HuAng, S., Asymptotic behavior of solutions of $u_{t}=\Delta \phi_{m}(u)$ as $m \rightarrow$ $\infty$ with inconsistent initial values. In Analyse Mathématique et applications, Gauthier-Villars, 1988. Zb10676.35041 MR0956958

28. Hardy, G. H. , Littlewood, J.E., \& Pólya, G.. Inequalities. Cambridge, at the University Press, 1952, 2nd ed. Zb10047.05302 MR0046395

29. IgBidA, N., The mesa-limit of the porous medium equation and the Hele-Shaw problem. Differential Integral Equations 15 (2002), 129-146. Zbl1011. 35080 MR1870466

30. De Pablo, A., Quirós, F., Rodríguez, A., \& VÁzQuez, J. L., A fractional porous medium equation. Adv. Math. 226 (2011), 1378-1409. Zbl1208. 26016 MR2737788

31. De Pablo, A., Quirós, F., Rodríguez, A., \& VÁzQuez, J. L., A general fractional porous medium equation. Comm. Pure Appl. Math. 65 (2012), 1242-1284. Zbl1248. 35220 MR2954615

32. LandKof, N.S., Foundations of modern potential theory, Die Grundlehren der mathematischen Wissenschaften, Band 180. Springer Verlag, New York Heidelberg, 1972. Zb10253. 31001 MR0350027

33. Pólya, G., \& SZEgö, C., Isoperimetric inequalities in Mathematical Physics. Princeton Univ. Press, no. 27, 1951. Zbl0044.38301 MR0043486

34. Quirós, F. , \& VÁzQuez, J.L., Mesa limit problems for the fractional porous media equation, in preparation.

35. Rodriguez, A., \& VAZquez, J. L., Obstructions to existence in fast-diffusion equations. J. Diff. Eqns. 184 (2002), 348-385. Zbl1007.35042 MR1929882

36. SACKS, P. E., A singular limit problem for the porous medium equation. J. Math. Anal. Appl. 140 (1989), 456-466. Zb10688.35035 MR1001869

37. Silvestre, L.E., Hölder estimates for solutions of integro differential equations like the fractional Laplace. Indiana Univ. Math. J. 55 (2006), 1155-1174. Zbl1101. 45004 MR2244602

38. Stan, D., DEL Teso, F., \& VÁzquez, J. L., Finite and infinite speed of propagation for porous medium equations with fractional pressure. Comptes Rendus Mathématique (Comptes Rendus Acad. Sci. Paris) 352 (2014), 123-128. Zbl1284.35352 MR3151879

39. Stan, D., Del Teso, F., \& VÁzQuez, J.L., Transformations of self-similar solutions for porous medium equations of fractional type. Nonlinear Anal., Theory Methods Appl. Ser. A, Theory Methods 119 (2015), 62-73. Zbl06446443 MR3334174

40. Stein, E. M.. Singular integrals and differentiability properties of functions. Princeton Mathematical Series, No. 30, Princeton University Press, Princeton, N.J. 1970.

41. Talenti, G., Elliptic equations and rearrangements. Ann. Scuola Norm. Sup. 3 (1976), 697-718. Zb10341.35031 MR0601601

42. Talenti, G., Nonlinear elliptic equations, rearrangements of functions and Orlicz spaces. Annal. Mat. Pura Appl. 120 (1979), 159-184. Zb10419. 35041 MR0551065

43. DEL TESO, F., Finite difference method for a fractional porous medium equation. Calcolo 51 (2014), 615-638. Zbl1310.76115 MR3279579 
44. Del Teso, F. , \& VÁzquez, J.L., Finite difference method for a general fractional porous medium equation. (2013) arXiv:1307.2474.

45. VÁzquez, J. L., Symétrisation pour $u_{t}=\Delta \varphi(u)$ et applications. C. R. Acad. Sc. Paris 295 (1982), 71-74. Zbl0501.35015 MR0676365

46. VÁzQUEZ, J. L., Nonexistence of solutions for nonlinear heat equations of fast-diffusion type. J. Math. Pures. Appl. 71 (1992), 503-526. Zb10694.35088 MR1193606

47. VÁzquez, J. L., Symmetrization and mass comparison for degenerate nonlinear parabolic and related elliptic equations. Advances in Nonlinear Studies 5 (2005), 87-131. Zbl1085 . 35083 MR2117623

48. VÁzQuez, J. L., The porous medium equation. Mathematical theory. Oxford Mathematical Monographs. The Clarendon Press, Oxford University Press, Oxford, 2007. ISBN: 978-0-19-856903-9. Zb11107.35003 MR2286292

49. VÁzQUEZ, J. L., Smoothing and Decay Estimates for Nonlinear Diffusion Equations. Equations of Porous Medium Type. Oxford Lecture Series in Mathematics and its Applications, 33. Oxford University Press, Oxford, 2006. Zbl1113.35004 MR2282669

50. VÁzquez, J.L., Nonlinear Diffusion with Fractional Laplacian Operators. In Nonlinear partial differential equations: the Abel Symposium 2010, Holden, Helge \& Karlsen, Kenneth H. (eds.), Springer, 2012. Pp. 271-298. Zbl1248.35223 MR3289370

51. VÁzQUEZ, J. L., Barenblatt solutions and asymptotic behaviour for a nonlinear fractional heat equation of porous medium type. J. Eur. Math. Soc. 16 (2014), 769-803. Zbl1297. 35279 MR3191976

52. VÁzQUEZ, J. L., Recent progress in the theory of nonlinear diffusion with fractional Laplacian operators. In Nonlinear elliptic and parabolic differential equations, Disc. Cont. Dyn. Syst. - S 7 (2014), 857-885. Zbl1290.26010 MR3177769

53. VÁZQUEZ, J. L., Existence of maximal solutions for some very singular nonlinear fractional diffusion equations in 1D. Preprint 2015. arXiv:1505.04902 [math.AP]

54. VÁzquez, J. L., \& Volzone, B., Symmetrization for linear and nonlinear fractional parabolic equations of porous medium type. J. Math. Pures Appl. 101 (2014), 553-582. Zbl1290 . 35317 MR3192423

55. VÁzQuez, J. L., \& Volzone, B., Optimal estimates for fractional Fast Diffusion Equations. J. Math. Pures Appl. 103 (2015), 535-556. Zb106390092 MR3298369

56. Weinberger, H., Symmetrization in uniformly elliptic problems. Studies in Math. Anal., Stanford Univ. Press, 1962, pp. 424-428. Zb10123.07202 MR0145191 